\title{
Flux tube model for glueballs
}

\author{
Masaharu Iwasaki, ${ }^{1, *}$ Shin-Ichi Nawa, ${ }^{2, \dagger}$ Takayoshi Sanada, ${ }^{1}$ and Fujio Takagi ${ }^{3, *}$ \\ ${ }^{1}$ Department of Physics, Kochi University, Kochi 780-8520, Japan \\ ${ }^{2}$ Faculty of Human Life and Environmental Science, Kochi Women's University, Kochi 780-8515, Japan \\ ${ }^{3}$ Department of Basic Science, Ishinomaki Senshu University, Ishinomaki 986-8580, Japan
}

(Received 2 May 2003; published 23 October 2003)

\begin{abstract}
We calculate the mass spectra and decay widths of glueballs in the flux tube model. The glueball is assumed to be a flux tube ring. The breathing and the rotational motions are investigated using the WKB approximation. The calculated spectrum is consistent with those obtained by lattice QCD. The decay widths are also computed using the Schwinger mechanism, and it is shown that they have rather large values.
\end{abstract}

DOI: 10.1103/PhysRevD.68.074007

PACS number(s): 12.39.Mk, 12.40.-y, 13.30.Eg

\section{INTRODUCTION}

It is widely believed that the strong interaction is described by quantum chromodynamics (QCD) [1]. It is, however, very difficult to solve QCD, so that several effective models have been devised to explain the physical properties of many hadrons. The flux tube model [2], or hadron string model [3,4], is one such model. According to this model, mesons are made up of a quark and antiquark connected by a color flux tube and baryons are described by a quark and diquark connected by the same flux tube. We showed in a previous paper [5] that various excited states of the hadrons can be explained systematically using such a simple model.

On the other hand, recent lattice QCD simulations [6] predict the existence of glueballs, which are composed of only gluons. It is the purpose of this paper to present an intuitive and analytic approach to glueballs using a flux tube model. We assume that the glueball is a closed color flux tube (flux tube ring). Moreover, the flux tube that constitutes the ring is assumed to have the same properties as the flux tube that constitutes a meson or a baryon. Consequently, our model has no free parameter. We study the relativistic motion of the closed string and obtain the mass spectrum of the glueballs. This simple picture has already been considered by several authors. In particular, Koma, Suganuma, and Toki [7] studied the relativistic breathing motion analytically with the use of the dual Ginzburg-Landau theory $[8-10]$. We study not only the vibrational motion but also the rotational one. Moreover, the decay widths in [7] are also calculated by taking into account $q \bar{q}$ pair production inside the flux tube (Schwinger mechanism).

This paper is organized as follows. In the next section, the flux tube model for the glueballs is formulated. Section III is devoted to calculation of the mass spectra and the decay widths of the glueballs. In Sec. IV, the numerical results are presented and some discussion is given.

\section{A STRING MODEL OF GLUEBALLS}

According to our flux tube model [5], mesons (baryons) are composed of a quark $q$ and an antiquark $\bar{q}$ (diquark $q q$ )

\footnotetext{
*Electronic address: miwasaki@cc.kochi-u.ac.jp

†Electronic address: nawa@cc.kochi-wu.ac.jp

*Electronic address: takagi@isenshu-u.ac.jp
}

connected by a color flux tube. The color quantum number of $\bar{q}$ is the same as that of $q q$ so that both flux tubes are identical. This fact means the slope of the Regge trajectories of the mesons and baryons is universal.

Now let us consider a flux tube configuration that corresponds to a glueball state. Since it is a quarkless color singlet state, we assume that the flux tube forms a closed circle (ring). Under this assumption, we will construct a mathematical expression for the flux tube model of the glueball. Let us start with the Lagrangian of the MIT bag model applied to a toruslike bag [11],

$$
L=\frac{1}{2} \int\left(\mathbf{H}^{2}-\mathbf{E}^{2}\right) d^{3} x-\int B d^{3} x,
$$

where $\mathbf{E}$ and $\mathbf{H}$ are the color electric and magnetic fields, respectively and the $B$ in the second term is the bag constant. We consider the breathing motion and the rotational motion around a diameter of the ring; a closed string with varying radius $r(t)$ rotates around the $x$ axis with angular velocity $\omega=\dot{\theta}(t)$ as shown in Fig. 1. The volume integral in the right-

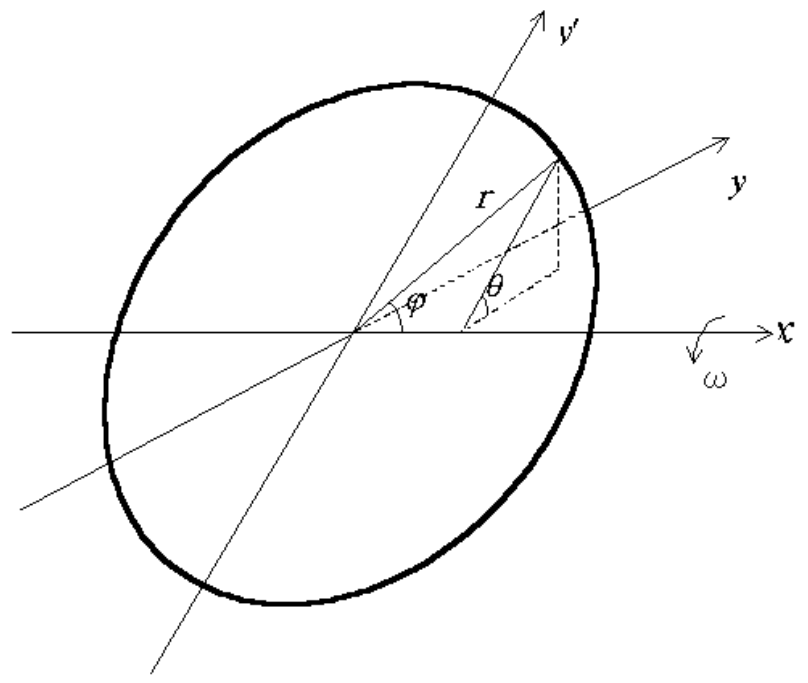

FIG. 1. A closed string with radius $r$ rotating around the $x$ axis with angular velocity $\omega$. 
hand side of Eq. (1) can be rewritten in a coordinate system that rotates together with the ring. If the color flux in the rotating coordinate is denoted by $\mathbf{E}_{0}$, we get $\mathbf{H}^{2}-\mathbf{E}^{2}$ $=-\mathbf{E}_{0}^{2}$. Then the Lagrangian is approximated by the line integral,

$$
L(r, \dot{r}, \omega)=-\oint\left(\frac{1}{2} \mathbf{E}_{0}^{2}+B\right) \Delta S_{0} \sqrt{1-v^{2}} d l,
$$

where the volume element of the flux tube with infinitesimal length $d l$ is approximated by $\Delta S_{0} \sqrt{1-v^{2}} d l . \Delta S_{0}$ means the cross section of the flux tube in the rotational coordinate system. The velocity $\mathbf{v}$ of the line element is perpendicular to the direction of the line element so that the factor $\sqrt{1-v^{2}}$ represents the Lorenz contraction. If the generalized coordinates of the line element are written as those in Fig. 1, the squared velocity is given by $v^{2}=\dot{r}^{2}+r^{2} \omega^{2} \sin ^{2} \varphi$. Consequently, we obtain the following Lagrangian of the flux tube ring:

$$
L(r, \dot{r}, \omega)=-\int_{0}^{2 \pi} \operatorname{ar} \sqrt{1-v^{2}} d \varphi
$$

where the constant $a$ means the string tension given by $a$ $\equiv\left(\mathbf{E}_{0}^{2} / 2+B\right) \Delta S_{0}$. Moreover, if we think of the pressure balance on the flux tube, we get $\mathbf{E}_{0}^{2} / 2=B$ [12].

Now let us quantize the Lagrangian in order to calculate the energy eigenvalues of the glueballs. The generalized coordinates of our system are the radius $r$ of the flux tube ring and the rotational angle $\theta$ of the ring around the $x$ axis. The conjugate momenta are defined by

$$
\begin{aligned}
& p_{r} \equiv \frac{\partial L}{\partial \dot{r}}=\int \frac{a r \dot{r}}{\sqrt{1-v^{2}}} d \varphi \equiv H \dot{r}, \\
& p_{\theta} \equiv \frac{\partial L}{\partial \omega}=\int \frac{a r^{3} \omega \sin ^{2} \varphi}{\sqrt{1-v^{2}}} d \varphi \equiv I \omega,
\end{aligned}
$$

where $p_{r}$ and $p_{\theta}$ represent the radial momentum and the angular momentum of the system. It will also be shown that the quantities $H$ and $I$ introduced in the right-hand side correspond to the energy and moment of inertia of the closed string, respectively. The Hamiltonian is given by

$$
H \equiv \dot{r} p_{r}+\omega p_{\theta}-L=\int \frac{a r}{\sqrt{1-v^{2}}} d \varphi .
$$

Canonical quantization leads to replacement of the momenta by the following operators:

$$
\begin{aligned}
& p_{r} \rightarrow \hat{p}=-i \hbar \frac{1}{r} \frac{\partial}{\partial r} r, \\
& p_{\theta} \rightarrow \hat{l}=-i \hbar \frac{\partial}{\partial \theta} .
\end{aligned}
$$

Thus we have the Schrödinger equation with the energy eigenvalue $E$,

$$
\hat{H}(r, \hat{p}, \hat{l}) \psi(r, \theta)=E \psi(r, \theta),
$$

and the normalization of the wave function is given by

$$
\int_{0}^{\infty} d r \int_{0}^{2 \pi} d \theta r^{2}|\psi(r, \theta)|^{2}=1 .
$$

If the wave function is rewritten as

$$
\psi(r, \theta)=\frac{1}{\sqrt{2 \pi}} \chi(r) e^{i l \theta / \hbar}
$$

and substituted into Eq. (9), we get

$$
\hat{H}(r, \hat{p}, l) \chi(r)=E \chi(r) .
$$

Here $l$ is an eigenvalue of the angular momentum, which is an even integer $(l=0,2,4, \ldots)$ because of the boundary condition $\psi(r, \theta=0)=\psi(r, \theta=2 \pi)$.

\section{THE EXCITED ENERGY AND THE DECAY WIDTH}

We will try to solve the Schrödinger equation (12) and calculate the energy spectrum and the decay width. In the case of $l=0$, we notice that $\omega=0$ from Eq. (5). Then the Schrödinger equation is reduced to

$$
\left\{\hat{p}^{2}+(2 \pi a r)^{2}\right\} \chi(r)=E^{2} \chi(r) .
$$

It should be noted that this equation is nothing but the Schrödinger equation of a three-dimensional harmonic oscillator. Introducing a new function $\rho(r)$ by $\chi(r) \equiv \rho(r) / r$, we have the Schrödinger equation for the one-dimensional harmonic oscillator, so that we obtain the familiar eigenvalues for $E^{2}$,

$$
E^{2}=2 \pi a \hbar(2 n+1)
$$

where the quantum number $n$ is an odd integer ( $n$ $=1,3,5, \ldots)$ from the boundary condition $\rho(0)=0$. This result is identical to that obtained by Koma, Suganuma, and Toki [7].

Next we consider the case of $l>0$. In order to solve the Schrödinger equation, we will extend the WKB approximation [13] to the relativistic equation. The wave function $\chi(r)$ is rewritten as

$$
\chi(r)=A(r) e^{i S(r) / \hbar},
$$

where the amplitude $A(r)$ and the phase $S(r)$ are unknown real functions. Substituting it into Eq. (12), we get

$$
\hat{H}\left(r, e^{-i S / \hbar} \hat{p} e^{i S / \hbar}, l\right) A(r)=E A(r) .
$$

Here we expand the left-hand side of this equation with respect to $\hbar$. The lowest order equation leads to

$$
H(r, p, l)=E,
$$

where $p$ stands for $d S / d r$. From this equation, we get the phase function $S(r)$ and the energy eigenvalue $E$. The next order of $\hbar$ gives 


$$
\frac{1}{2}\left(\frac{\partial H}{\partial p} \hat{p}+\hat{p} \frac{\partial H}{\partial p}\right) A(r)=0,
$$

where the operators on the left-hand side have been symmetrized because it should be Hermitian. With a simple calculation, this equation can be transformed into

$$
\frac{d}{d r}\left(\frac{\partial H}{\partial p} r^{2} A\right)=0
$$

By integrating this equation we obtain the amplitude function $A(r)$. The integration constant should be determined by the normalization condition of the wave function. Thus we have obtained the wave function of the glueball.

First we calculate the exited spectra of the glueball. There are two constants of motion,

$$
\begin{gathered}
E=\frac{4 a r}{\sqrt{1-(p / E)^{2}}} K_{1}(k), \\
l=\frac{4 a r^{2}}{k}\left[K_{1}(k)-K_{2}(k)\right],
\end{gathered}
$$

where $K_{1}(k)$ and $K_{2}(k)$ are the complete elliptic integrals of the first and second kinds defined by

$$
\begin{aligned}
& K_{1}(k) \equiv \int_{0}^{\pi / 2} \frac{1}{\sqrt{1-k^{2} \sin ^{2} \varphi}} d \varphi, \\
& K_{2}(k) \equiv \int_{0}^{\pi / 2} \sqrt{1-k^{2} \sin ^{2} \varphi} d \varphi .
\end{aligned}
$$

Here $k(0 \leqslant k<1)$ is defined by

$$
k \equiv \frac{r \omega}{\sqrt{1-(p / E)^{2}}} .
$$

If Eq. (20) is squared, the energy is represented as

$$
E^{2}=p^{2}+16 a^{2} r^{2} K_{1}(k)^{2} \equiv p^{2}+V^{2} .
$$

The $V$ in the right-hand side means the scalar potential of the closed string and is written as

$$
V(r) \equiv 4 a K_{1}(k) r=\frac{k l}{r}+4 a K_{2}(k) r .
$$

It should be noted that the potential is not at all a linear function of $r$, because $k$ in this equation is a function of $r$ through Eq. (21). The excited spectra can be calculated using the Bohr-Sommerfeld formula:

$$
\oint p(r) d r=(2 n+1) 2 \pi \hbar .
$$

If Eq. (25) is substituted into the left-hand side of this equation, the energy $E$ is determined $(n=1,3,5, \ldots)$.

Next let us discuss the decay widths of the glueballs. To this end, we must introduce a new interaction in our present model. We assume that the pair creation of $q \bar{q}$ in the flux tube leads to the decay of the closed string: string $\rightarrow q \bar{q} \rightarrow$ many hadrons. This mechanism, which is called the Schwinger mechanism [14], was used by us to calculate the decay widths of mesons and baryons in Ref. [3]. The probability of pair production in a unit space-time volume in the flux tube is given by

$$
w=\frac{a^{2}}{4 \pi^{3}} \sum_{q} \sum_{n=1}^{\infty} \frac{1}{n^{2}} \exp \left(-\frac{n \pi m_{q}^{2}}{a}\right),
$$

where $a$ is the string tension and $\Sigma_{q}$ indicates a summation over all quark flavors with mass $m_{q}(q=u, d, s)$ [15-17]. The probability of decay (pair production) in the time interval $d t$ is given by

$$
d W=\int|\chi(r)|^{2} w \Omega(r) r^{2} d r d t
$$

where $\Omega(r)$ denotes the volume of the flux tube ring with radius $r$ and is given by

$$
\Omega(r)=\Delta S_{0} \int_{0}^{2 \pi} \sqrt{1-v^{2}} r d \varphi .
$$

Substituting this equation into Eq. (28), we obtain the decay width

$$
\Gamma \equiv \frac{d W}{d t}=\int_{0}^{\infty} 4 b r^{3} \sqrt{1-\left(\frac{p}{E}\right)^{2}} K_{2}(k) A^{2} d r .
$$

The constant $b=w \Delta S_{0}$ defined in the right-hand side means the probability of the string breaking per unit length per unit time.

\section{NUMERICAL RESULTS AND DISCUSSION}

In this section we will carry out numerical calculations of the mass spectra and the decay widths of glueballs. There are two parameters in our model: the string tension $a$ and the string breaking probability $b$. It is assumed that the color flux tube that constitutes a glueball is the same as the one that constitutes a meson. The reason is that the color flux from a quark (antiquark) is the narrowest one in the hadron world. Thus the two parameters are taken to have the same values as those used in our previous paper [5], which reproduce the string tension $a=0.15 \mathrm{GeV}^{2}$ and the decay width $\Gamma_{\rho}$ $=151 \mathrm{MeV}$.

Before carrying out the calculations, it is instructive to figure out the potential $V(r)$ as a function of $r$. From Eq. (26), the potentials with various angular momenta are drawn in Fig. 2. A repulsive core appears near the origin, due to the centrifugal potential. In contrast, the potential becomes linear at larger distances. This behavior is natural and can be understood analytically as follows. When $r \rightarrow 0$, we note that $k \rightarrow 1$ from Eq. (21). Then the potential is reduced to the centrifugal one: $E \rightarrow \sqrt{p^{2}+(l / r)^{2}} \approx p+l^{2} /\left(2 p r^{2}\right)$. On the other hand, it approaches the linear potential at larger distances, which is the confining potential: $V(r) \rightarrow 2 \pi a r$. 


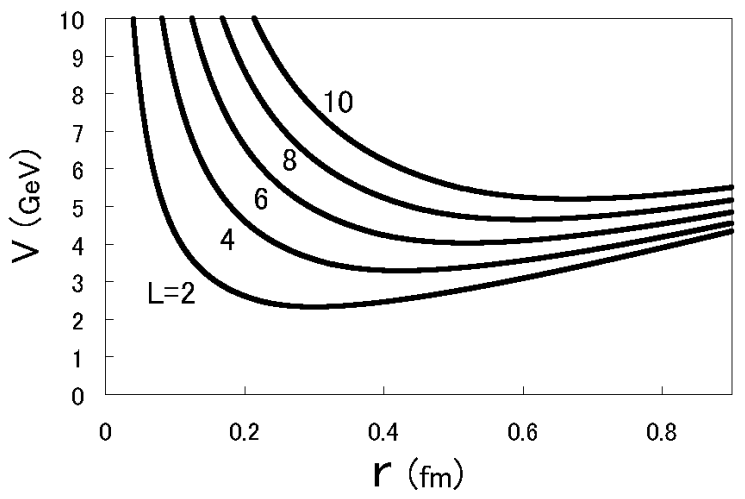

FIG. 2. The potential for the breathing mode. The $L$ in the figure denotes the angular momentum of the closed string.

The mass spectrum of the vibrational mode has a simple form: the vibrational quantum number $(n=1,3,5, \cdot)$ is proportional to the squared mass from Eq. (14). The relation between the quantum number and the squared mass is an analogue of the Regge trajectory of rotational motion. The vibrational modes on the straight line correspond to the $l$ $=0$ states that lie on the (Regge) parent and (many) daughter trajectories with equal spacing.

On the other hand, the spectrum of the rotational mode is shown in Fig. 3. The horizontal axis is the angular momentum $(L=0,2,4, \ldots)$. The mass spectrum in Fig. 3 is drawn in the case of $n=0$ where the boundary condition at $r=0$ does not exist due to the centrifugal potential. We obtained an almost straight line: $M^{2} \propto L$. The Regge slope $d L / d M^{2}$ is smaller than that of the ordinary Regge trajectories of mesons and baryons. This difference seems to originate from the geometrical structure of the string. If the total length of the string is fixed, the moment of inertia of the ring is smaller than that of a sticklike string (meson and baryons). On the other hand, the energy of the string is proportional to the total length. Therefore the slope of the Regge trajectory of the glueball is smaller than that of ordinary hadrons.

The calculated decay widths are shown by the closed circles in Fig. 4 and Fig. 5 for the vibrational and rotational modes, respectively. They are considerably larger than those

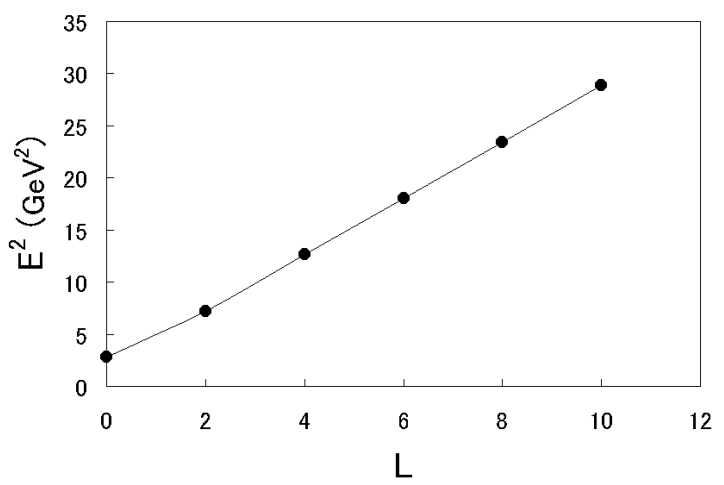

FIG. 3. The mass spectrum of the rotational mode as a function of the angular momentum.

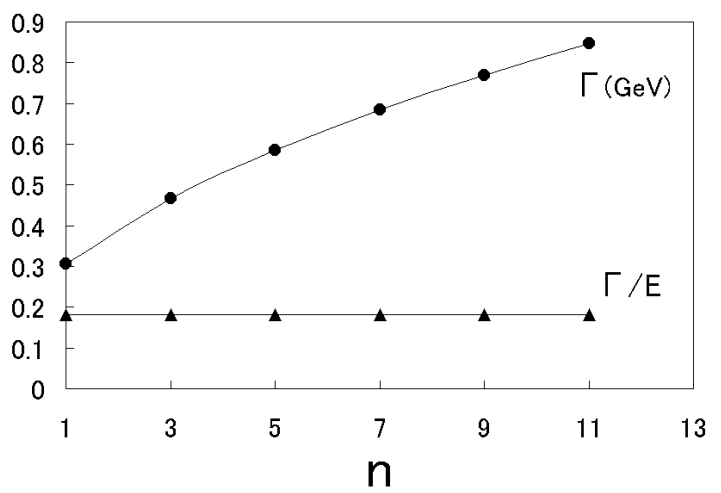

FIG. 4. The decay width of the vibrational mode as a function of the vibrational quantum number. The triangles in the figure denote the ratio of the decay width to the mass.

of ordinary hadrons, so that glueballs generally decay easily. This fact explains why no glueballs have been found experimentally. The triangles in the same figures represent the ratio of the decay width to the mass. In the case of the vibrational mode (Fig. 4), the decay widths are proportional to their masses exactly as shown below. The constancy of the ratio seems to hold also for the rotational mode, as seen in Fig. 5.

In the case of $l=0$, the decay width has a simple relation to the excited energy. Noting that $K_{2}(k=0)=\pi / 2$ since $\omega$ $=0$, Eq. (31) is transformed into

$$
\Gamma=\frac{4 \pi^{2} a b}{E} \int_{0}^{\infty} A^{2} r^{4} d r=\frac{4 \pi^{2} a b}{E}\left\langle r^{2}\right\rangle .
$$

The $\left\langle r^{2}\right\rangle$ denotes the expectation value for the excited state. Thus we have

$$
\Gamma=\frac{b}{2 a} E,
$$

where $E$ is the energy of the corresponding excited state. The relation can be understood as follows. The mass of a hadron is proportional to its length. On the other hand, the breaking

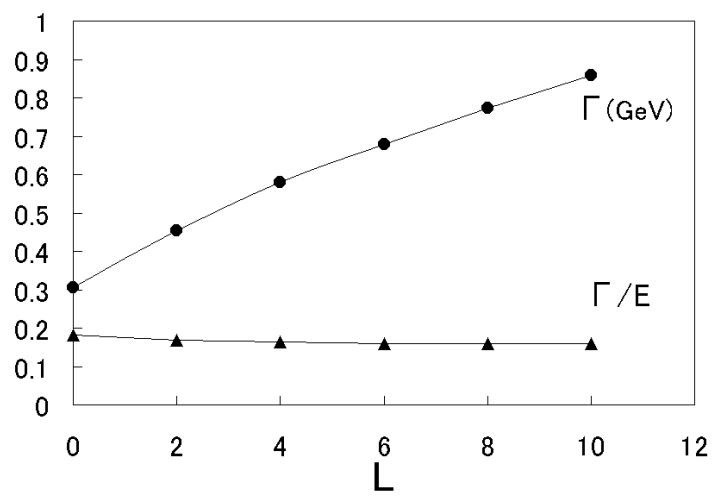

FIG. 5. The decay width of the rotational mode as a function of the angular momentum. The triangles in the figure denote the ratio of the decay width to the mass. 
TABLE I. Mass spectrum of the glueballs. They are calculated by lattice QCD and by our model.

\begin{tabular}{lcc}
\hline \hline & Lattice QCD $(\mathrm{MeV})$ & Our model $(\mathrm{MeV})$ \\
\hline $0_{1}$ & 1.7 & 1.68 \\
$0_{2}$ & $2.5-2.8$ & 2.57 \\
$2_{1}$ & 2.4 & 2.69 \\
\hline \hline
\end{tabular}

probability is also proportional to the length because the cross section of the tube is the same for all hadrons (universality). Therefore we obtain the linearity $\Gamma \propto E$.

Finally, we add some comments on the experimental data [18]. There are some candidates: $f_{0}(1500)$ and $f_{0}(1710)$ for the scalar glueball; $f_{2}(2300)$ and $f_{2}(2340)$ for the tensor glueball. Unfortunately, the abundance of $q-\bar{q}$ meson states in the $1-3 \mathrm{GeV}$ region and the possibility of quarkoniumglueball mixing states still make it difficult to identify the glueball states. On the other hand, the glueballs have been "found" on a lattice [19-25]. Here we refer to the results obtained by lattice QCD simulation [6], which are shown in Table I. The first and second $J=0$ states are expected to be the breathing modes of the glueball. These values are consis- tent with those obtained in our model. The first $J=2$ state is the glueball state by the rotational motion and the energy is slightly smaller than that obtained in our model. This may be due to the assumption that the shape of the ring is always circular. A centrifugal force would deform the shape so that the ring would become elliptic. If this effect is taken into account, the moment of inertia of the ring becomes large and the excitation energy is lowered.

In conclusion, we have developed a flux tube model of glueballs as well as mesons and baryons. The glueball is described by a flux tube ring (closed string), which is characterized by two parameters, the string tension and the string breaking parameter. They are the same as those in the case of mesons and baryons. The mass spectra calculated by our model are consistent with those of lattice QCD. The decay widths are several hundreds of $\mathrm{MeV}$. These values are rather large, and it is desirable to compute the decay widths definitively by lattice QCD [23-25].

\section{ACKNOWLEDGMENTS}

We would like to thank the Nuclear Theory Group at Kochi University for helpful discussions. In particular, we would like to thank Yuma Harada for his help with the numerical calculations in the early stages of this work.
[1] F. Wilczek, Annu. Rev. Nucl. Part. Sci. 32, 177 (1982).

[2] N. Isgur and J. Paton, Phys. Lett. 124B, 247 (1983).

[3] A.B. Migdal, S.B. Khokhlachev, and V.Yu. Borue, Phys. Lett. B 228, 167 (1989).

[4] A.B. Migdal, Nucl. Phys. A518, 358 (1990).

[5] M. Iwasaki and F. Takagi, Phys. Rev. D 59, 094024 (1999).

[6] C. Morningstar and M. Peardon, Phys. Rev. D 60, 034509 (1999).

[7] Y. Koma, H. Suganuma, and H. Toki, Phys. Rev. D 60, 074024 (1999).

[8] T. Suzuki, Prog. Theor. Phys. 80, 929 (1988).

[9] S. Maedan and T. Suzuki, Prog. Theor. Phys. 81, 229 (1989).

[10] H. Suganuma, S. Sasaki, and H. Toki, Nucl. Phys. B435, 207 (1995).

[11] A. Chodos, R.L. Jaffe, K. Johnson, C.B. Thorn, and V. Weisskopf, Phys. Rev. D 9, 3471 (1974).

[12] K. Johnson and C.B. Thorn, Phys. Rev. D 13, 1934 (1976).

[13] A.B. Migdal and V.P. Krainov, Approximation Methods in Quantum Mechanics (W.A. Benjamin, New York, 1969).

[14] J. Schwinger, Phys. Rev. 82, 664 (1951).
[15] E.G. Gurvich, Phys. Lett. 87B, 386 (1979).

[16] A. Casher, H. Neuberger, and S. Nussinov, Phys. Rev. D 20, 179 (1979).

[17] N.K. Glendenning and T. Matsui, Phys. Rev. D 28, 2890 (1983).

[18] Particle Data Group, K. Hagiwara et al., Phys. Rev. D 66, 010001 (2002).

[19] G. Bali, K. Schilling, A. Hulsebos, A. Irving, C. Michael, and P. Stephenson, Phys. Lett. B 309, 378 (1993).

[20] H. Chen, J. Sexton, A. Vaccarino, and D. Weingarten, Nucl. Phys. B (Proc. Suppl.) 34, 357 (1994).

[21] J. Sexton, A. Vaccarino, and D. Weingarten, Phys. Rev. Lett. 75, 4563 (1995).

[22] J. Sexton, A. Vaccarino, and D. Weingarten, Nucl. Phys. B (Proc. Suppl.) 47, 128 (1996).

[23] M. Boglione and M.R. Pennington, Phys. Rev. Lett. 79, 1998 (1997).

[24] J. Cao, T. Huang, and H. Wu, Phys. Rev. D 57, 4154 (1998).

[25] C. Morningstar and M. Peardon, Phys. Rev. D 56, 4043 (1997). 